\title{
Rumen fermentation kinetics as impacted by red amaranth (Amaranthus cruentus, $L$ ) leaf powder using in vitro gas production technique
}

Thiwakorn Ampapon

Department of Animal Science, Rajamangala University of Technology Isan, Surin Campus.

\section{Bounnaxay Viennasay}

Department of Animal Science, Khon Kaen University.

Metha Wanapat ( $\nabla$ metha@kku.ac.th )

Khon Kaen University https://orcid.org/0000-0002-7633-052X

Research article

Keywords: phytonutrient, rumen enhancer, fermentation

Posted Date: April 17th, 2020

DOI: https://doi.org/10.21203/rs.3.rs-20200/v1

License: (1) This work is licensed under a Creative Commons Attribution 4.0 International License.

Read Full License 


\section{Abstract \\ Background}

A need for research searching for alternative rumen enhancers warrants immediate attention. The in vitro fermentation experiment was conducted using factorial arrangement of two factors of roughage to concentrate and seven level of red amaranth leaf powder percentage of total substrate in a Completely randomized design (CRD). Two factors, namely Factor $A$ was two ratio of roughage (R) to concentrate (C) at 60:40 and 40:60 and Factor B was level of red amaranth (Amaranthus cruentus, $L$ ) leaf powder (RALP) supplementation at $0,2,4,6,8,10$, and $12 \%$ of total dietary substrate.

\section{Results}

Red amaranth leaf powder (RALP) contained phytonutrients both condensed tannins and saponins in addition with high macro minerals $(\mathrm{Ca}, \mathrm{K}$, and $\mathrm{Mg}$ ). This experiment revealed innovations of the RALP supplementation by enhancing rumen propionate $\left(C_{3}\right)$ production, reducing acetate $\left(C_{2}\right)$ to $\left(C_{3}\right)$ ratio, reducing protozoal population and mitigating methane $\left(\mathrm{CH}_{4}\right)$ production. Furthermore, rumen dry matter degradation percentages were remarkably enhanced $(P<0.001)$ by increasing RALP supplementation.

\section{Conclusion}

Plants rich in phytonutrients and minerals such as red amaranth leaf powder (RALP) have a vital and promising role in modulating rumen fermentation, mitigating methane production, as well as increasing substrate degradability.

\section{Background}

"Feeding the bugs, feeding the cows" has been stated for a long time in order to provide the rumen to perform the anaerobic fermentation process yielding the fermentation end-products degraded by residing microbiomes [1]. Wolin [2] and Murphy et al. [3] profoundly reported the stoichiometric balance in the rumen, showing the occurrence of rumen methane production via the metabolic pathway of rumen acetic production while capturing it in the propionate production randomization pathway. Using chemicals and/or antibiotics as rumen buffers or regulators have resulted improvement of rumen fermentation efficiency by increasing propionate while mitigating methane production [4]. Rumen $\mathrm{pH}$ has been demonstrated to greatly influence rumen microorganisms and their fermentation end-products $[5,6]$. By shifting levels of concentrate ratio, as well as supplementation, the rumen $\mathrm{pH}$ was dramatically reduced. Buffering rumen $\mathrm{pH}$ is therefore a necessity when the ruminants receiving high level of concentrate and supplementation. Herremans et al. [7], currently reviewed using a meta-analysis, confirming the beneficial effect of dietary tannins especially condensed tannins on improving the nitrogen utilization in ruminants 
by decreasing rumen protein degradation, ammonia-nitrogen concentration, blood urea-nitrogen, milk urea-nitrogen, whilst digestibility of protein was significantly lowered.

Feed resources and plant extracts containing phytonutrients (PTN) have been increasingly important, as they exerted impacts on rumen microorganisms and fermentation especially the effects of condensed tannins (CT) and saponins (SP) [8]. Many kinds of plants and fruit wastes, containing PTN have been shown with their impacts on modulating the rumen fermentation especially mitigating methane production [9-11].

Amaranth, namely red amaranth (Amaranthus cruentus, L.) have been revealed to contain high concentration of phytonutrients, in which they could exert significant effect on human health [12]. Amaranth (Amaranth spp.) is a nutritious crop containing high levels of crude protein, minerals, vitamins, as well as polyphenols and flavonoids especially in the leaf and seeds $[13,14]$. The main phenolic compounds reported in both leaf and seeds were gallic acid, vanillic acid and p-coumaric acid etc. [15]. Earlier study by Chairatanayuth [16] who found A. Spp. leaf especially in A. cruentus L., contained higher CP (16.50\%) lower NDF (41.50\%), when harvested at 45 days of growth. Khandaker et al. [17] reported the efficacy of polyphenols of red amaranth $(A$. trocor $L$.) on the antioxidant activity and have found that high level of total polyphenols in leaf was closely correlated $\left(P<0.05, \mathrm{R}^{2}=0.82\right)$ with the antioxidant activity. Nevertheless, no research work using red amaranth leaf on its influence on rumen fermentation characteristics and nutrients digestibility in ruminants. Therefore, this experiment aimed it investigating the effect of red amaranth (Amaranthus cruentus, L.) on in vitro fermentation gas production experiment.

\section{Results}

Table 1, presents details of feeds and their nutritive values. Rice straw composited of $2.80 \% \mathrm{CP}, 72.70 \%$ NDF and $47.50 \%$ ADF, respectively. Concentrate was formulated and analyzed to contain $14.40 \% \mathrm{CP}, 75 \%$ TDN, $28.90 \%$ NDF, and $17.20 \%$ ADF. The nutritive value of red amaranth leaf power were $15.80 \% \mathrm{CP}$, $40.30 \% \mathrm{NDF}, 29.40 \% \mathrm{ADF}$ and $2.37 \% \mathrm{Ca}, 2.33 \% \mathrm{~K}$, and with $0.90 \%$ condensed tannins, $0.50 \%$ saponins, respectively. 
Table 1

Feed ingredients and their chemical compositions

\begin{tabular}{|c|c|c|c|}
\hline Items & Rice straw & Concentrate & Red amaranth leaf powder (RALP) \\
\hline \multicolumn{4}{|c|}{ Ingredients (\% of Fresh basis) } \\
\hline Cassava chip & 0.00 & 60.00 & 0.00 \\
\hline Brewers' grain (dried) & 0.00 & 12.00 & 0.00 \\
\hline Rice bran & 0.00 & 9.00 & 0.00 \\
\hline Palm kernel meal & 0.00 & 13.00 & 0.00 \\
\hline Urea & 0.00 & 2.00 & 0.00 \\
\hline Molasses & 0.00 & 2.00 & 0.00 \\
\hline Sulfur & 0.00 & 0.50 & 0.00 \\
\hline Salt & 0.00 & 1.00 & 0.00 \\
\hline Mineral premix & 0.00 & 0.50 & 0.00 \\
\hline \multicolumn{4}{|c|}{ Chemical composition (\% DM) } \\
\hline Dry matter & 90.00 & 88.50 & 88.90 \\
\hline Crude protein & 2.80 & 14.40 & 15.80 \\
\hline Organic matter & 96.40 & 95.20 & 96.50 \\
\hline Neutral detergent fiber & 72.70 & 28.90 & 40.30 \\
\hline Acid detergent fiber & 47.50 & 17.20 & 29.40 \\
\hline Condensed tannins & 0.00 & 0.00 & 0.90 \\
\hline Saponins & 0.00 & 0.00 & 0.50 \\
\hline $\mathrm{TDN}^{*}$ & 0.00 & 75.00 & 0.00 \\
\hline \multicolumn{4}{|l|}{ Mineral (\%) } \\
\hline $\mathrm{Ca}$ & 0.00 & 0.00 & 2.37 \\
\hline K & 0.00 & 0.00 & 2.33 \\
\hline $\mathrm{Mg}$ & 0.00 & 0.00 & 0.55 \\
\hline $\mathrm{Zn}$ & 0.00 & 0.00 & 0.08 \\
\hline $\mathrm{Fe}$ & 0.00 & 0.00 & 0.97 \\
\hline
\end{tabular}


The gas production kinetics parameters are presented in Table 2. Ratio of roughage to concentrate and percentage of red amaranth leaf powder (RALP) had impacted on the fermentation process and subsequently on the gas production kinetics $(b, c, a+b)$. The $c$ and $a+b$ values for both R:C ratio and RALP were highly significant. As level of RALP supplementation increased, regardless of R:C, the values were enhanced. For in vitro DM degradabilities percentages for both at 12 and $24 \mathrm{~h}$ the data were also incremental and were highly significant for both R:C and level of RALP supplementation. 
Table 2

Effect of red amaranth (Amaranth cruentus, L) leaf powder (RALP) supplementation on gas production and in vitro dry matter degradability (\%)

\begin{tabular}{|c|c|c|c|c|c|c|c|c|c|}
\hline \multirow[t]{2}{*}{ Treatments } & \multirow[t]{2}{*}{$\mathrm{R}: \mathrm{C}^{\mathrm{a}}$} & \multirow[t]{2}{*}{ RALPb } & \multicolumn{5}{|c|}{ Gas production kinetics ${ }^{c}(\mathrm{ml})$} & \multicolumn{2}{|c|}{$\begin{array}{l}\text { In vitro dry matter } \\
\text { degradability (\%) }\end{array}$} \\
\hline & & & $a$ & $b$ & $c$ & $a+b$ & Gas $^{d}$ & 12 & 24 \\
\hline T1 & $60: 40$ & 0 & $\begin{array}{l}-7.44 \\
\pm \\
0.06\end{array}$ & $\begin{array}{l}75.41 \\
\pm 0.31\end{array}$ & $\begin{array}{l}0.37 \\
\pm \\
0.001\end{array}$ & $\begin{array}{l}68.01 \\
\pm 1.51\end{array}$ & $\begin{array}{l}68.41 \\
\pm 1.51\end{array}$ & $\begin{array}{l}41.92 \pm \\
1.61\end{array}$ & $\begin{array}{l}43.71 \pm \\
1.21\end{array}$ \\
\hline $\mathrm{T} 2$ & $60: 40$ & 2 & $\begin{array}{l}-7.52 \\
\pm \\
0.08\end{array}$ & $\begin{array}{l}76.21 \\
\pm 1.14\end{array}$ & $\begin{array}{l}0.37 \\
\pm \\
0.001\end{array}$ & $\begin{array}{l}68.73 \\
\pm 1.62\end{array}$ & $\begin{array}{l}69.12 \\
\pm 1.61\end{array}$ & $\begin{array}{l}43.91 \pm \\
0.62\end{array}$ & $\begin{array}{l}46.32 \pm \\
1.72\end{array}$ \\
\hline T3 & $60: 40$ & 4 & $\begin{array}{l}-7.42 \\
\pm \\
0.01\end{array}$ & $\begin{array}{l}76.22 \\
\pm 0.19\end{array}$ & $\begin{array}{l}0.37 \\
\pm \\
0.001\end{array}$ & $\begin{array}{l}68.82 \\
\pm 1.03\end{array}$ & $\begin{array}{l}69.11 \\
\pm 1.02\end{array}$ & $\begin{array}{l}46.54 \pm \\
1.81\end{array}$ & $\begin{array}{l}48.72 \pm \\
1.61\end{array}$ \\
\hline T4 & $60: 40$ & 6 & $\begin{array}{l}-7.61 \\
\pm \\
0.03\end{array}$ & $\begin{array}{l}77.72 \\
\pm 1.24\end{array}$ & $\begin{array}{l}0.37 \\
\pm \\
0.001\end{array}$ & $\begin{array}{l}70.11 \\
\pm 1.22\end{array}$ & $\begin{array}{l}70.52 \\
\pm 1.24\end{array}$ & $\begin{array}{l}47.61 \pm \\
10.82\end{array}$ & $\begin{array}{l}51.34 \pm \\
0.22\end{array}$ \\
\hline T5 & $60: 40$ & 8 & $\begin{array}{l}-7.72 \\
\pm \\
0.03\end{array}$ & $\begin{array}{l}79.01 \\
\pm 1.25\end{array}$ & $\begin{array}{l}0.37 \\
\pm \\
0.001\end{array}$ & $\begin{array}{l}71.21 \\
\pm 1.31\end{array}$ & $\begin{array}{l}71.61 \\
\pm 1.32\end{array}$ & $\begin{array}{l}48.32 \pm \\
0.24\end{array}$ & $\begin{array}{l}52.32 \pm \\
1.51\end{array}$ \\
\hline T6 & $60: 40$ & 10 & $\begin{array}{l}-7.81 \\
\pm \\
0.03\end{array}$ & $\begin{array}{l}79.54 \\
\pm 0.18\end{array}$ & $\begin{array}{l}0.37 \\
\pm \\
0.001\end{array}$ & $\begin{array}{l}71.72 \\
\pm 0.54\end{array}$ & $\begin{array}{l}72.14 \\
\pm 0.51\end{array}$ & $\begin{array}{l}48.83 \pm \\
1.82\end{array}$ & $\begin{array}{l}55.41 \pm \\
1.52\end{array}$ \\
\hline T7 & $60: 40$ & 12 & $\begin{array}{l}-7.92 \\
\pm \\
0.07\end{array}$ & $\begin{array}{l}80.03 \\
\pm 1.24\end{array}$ & $\begin{array}{l}0.37 \\
\pm \\
0.001\end{array}$ & $\begin{array}{l}72.14 \\
\pm 1.62\end{array}$ & $\begin{array}{l}72.52 \\
\pm 1.64\end{array}$ & $\begin{array}{l}50.52 \pm \\
1.61\end{array}$ & $\begin{array}{l}54.62 \pm \\
1.11\end{array}$ \\
\hline T8 & $40: 60$ & 0 & $\begin{array}{l}-7.22 \\
\pm \\
0.04\end{array}$ & $\begin{array}{l}71.51 \\
\pm 1.67\end{array}$ & $\begin{array}{l}0.38 \\
\pm \\
0.001\end{array}$ & $\begin{array}{l}64.42 \\
\pm 1.32\end{array}$ & $\begin{array}{l}64.71 \\
\pm 1.62\end{array}$ & $\begin{array}{l}50.31 \pm \\
1.22\end{array}$ & $\begin{array}{l}56.31 \pm \\
0.93\end{array}$ \\
\hline T9 & $40: 60$ & 2 & $\begin{array}{l}-7.71 \\
\pm \\
0.02\end{array}$ & $\begin{array}{l}76.31 \\
\pm 1.56\end{array}$ & $\begin{array}{l}0.38 \\
\pm \\
0.001\end{array}$ & $\begin{array}{l}68.63 \\
\pm 1.71\end{array}$ & $\begin{array}{l}69.01 \\
\pm 1.21\end{array}$ & $\begin{array}{l}52.24 \pm \\
1.01\end{array}$ & $\begin{array}{l}57.43 \pm \\
0.94\end{array}$ \\
\hline T10 & $40: 60$ & 4 & $\begin{array}{l}-8.23 \\
\pm \\
0.09\end{array}$ & $\begin{array}{l}78.43 \\
\pm 0.33\end{array}$ & $\begin{array}{l}0.38 \\
\pm \\
0.001\end{array}$ & $\begin{array}{l}70.22 \\
\pm 1.32\end{array}$ & $\begin{array}{l}70.62 \\
\pm 1.74\end{array}$ & $\begin{array}{l}52.72 \pm \\
0.42\end{array}$ & $\begin{array}{l}57.41 \pm \\
0.71\end{array}$ \\
\hline T11 & $40: 60$ & 6 & $\begin{array}{l}-8.23 \\
\pm \\
0.11\end{array}$ & $\begin{array}{l}79.33 \\
\pm 1.54\end{array}$ & $\begin{array}{l}0.38 \\
\pm \\
0.001\end{array}$ & $\begin{array}{l}71.04 \\
\pm 0.32\end{array}$ & $\begin{array}{l}71.44 \\
\pm 0.32\end{array}$ & $\begin{array}{l}54.21 \pm \\
0.14\end{array}$ & $\begin{array}{l}61.52 \pm \\
0.81\end{array}$ \\
\hline T12 & $40: 60$ & 8 & $\begin{array}{l}-8.34 \\
\pm \\
0.11\end{array}$ & $\begin{array}{l}79.81 \\
\pm 1.35\end{array}$ & $\begin{array}{l}0.38 \\
\pm \\
0.001\end{array}$ & $\begin{array}{l}71.62 \\
\pm 1.44\end{array}$ & $\begin{array}{l}72.01 \\
\pm 1.41\end{array}$ & $\begin{array}{l}57.32 \pm \\
0.42\end{array}$ & $\begin{array}{l}62.23 \pm \\
0.63\end{array}$ \\
\hline
\end{tabular}




\begin{tabular}{|c|c|c|c|c|c|c|c|c|c|}
\hline \multirow[t]{2}{*}{ Treatments } & \multirow[t]{2}{*}{$\mathrm{R}: \mathrm{C}^{\mathrm{a}}$} & \multirow[t]{2}{*}{ RALPb } & \multicolumn{5}{|c|}{ Gas production kinetics ${ }^{c}(\mathrm{ml})$} & \multicolumn{2}{|c|}{$\begin{array}{l}\text { In vitro dry matter } \\
\text { degradability (\%) }\end{array}$} \\
\hline & & & $a$ & $b$ & $c$ & $a+b$ & Gas $^{d}$ & 12 & 24 \\
\hline T13 & $40: 60$ & 10 & $\begin{array}{l}-8.51 \\
\pm \\
0.04\end{array}$ & $\begin{array}{l}80.83 \\
\pm 0.72\end{array}$ & $\begin{array}{l}0.38 \\
\pm \\
0.001\end{array}$ & $\begin{array}{l}72.33 \\
\pm 1.72\end{array}$ & $\begin{array}{l}72.72 \\
\pm 1.72\end{array}$ & $\begin{array}{l}58.62 \pm \\
0.32\end{array}$ & $\begin{array}{l}62.22 \pm \\
0.83\end{array}$ \\
\hline T14 & $40: 60$ & 12 & $\begin{array}{l}-8.83 \\
\pm \\
0.14\end{array}$ & $\begin{array}{l}82.12 \\
\pm 1.11\end{array}$ & $\begin{array}{l}0.38 \\
\pm \\
0.001\end{array}$ & $\begin{array}{l}73.32 \\
\pm 0.71\end{array}$ & $\begin{array}{l}73.74 \\
\pm 1.71\end{array}$ & $\begin{array}{l}59.11 \pm \\
0.21\end{array}$ & $\begin{array}{l}63.81 \pm \\
0.77\end{array}$ \\
\hline \multicolumn{10}{|l|}{$P$-value } \\
\hline $\mathrm{R}: \mathrm{C}$ ratio & & & 0.05 & 0.87 & 0.001 & 0.001 & 0.93 & 0.0001 & 0.0001 \\
\hline RALP & & & 0.32 & 0.32 & 0.001 & 0.001 & 0.32 & 0.0001 & 0.0001 \\
\hline Interactions & & & 0.87 & 0.89 & 0.12 & 0.12 & 0.88 & 0.38 & 0.16 \\
\hline \multicolumn{10}{|c|}{$\begin{array}{l}\text { a Roughage : Concentrate ratio at } 60: 40 \text {, and } 40: 60 \% \\
\text { b Levels of red amaranth leaf powder (RALP) supplementation at } 0,2,4,6,8,10 \text {, and } 12 \% \text { of total } \\
\text { substrate } \\
\text { ca: the gas production from the immediately soluble fraction; } b \text { : the gas production from the insoluble } \\
\text { fraction; } c \text { : the gas production rate constant for the insoluble fraction; } a+b \text { : the gas potential extent of } \\
\text { gas production. } \\
\text { d: Cumulative gas production at } 96 \mathrm{~h}(\mathrm{ml} / 0.20 \mathrm{~g} \text { DM substrate); RALP : red amaranth leaf powder }\end{array}$} \\
\hline
\end{tabular}


Table 3 Effect of red amaranth (Amaranth cruentus, L) leaf powder (RALP) supplementation on rumen fermentation characteristics

\begin{tabular}{|c|c|c|c|c|c|c|c|c|c|}
\hline \multirow[t]{3}{*}{ Treatments } & \multirow[t]{3}{*}{$\mathrm{R}: \mathrm{C}^{\mathrm{a}}$} & \multirow[t]{3}{*}{ RALP ${ }^{b}$} & \multirow[t]{3}{*}{$\mathrm{pH}$} & \multirow{3}{*}{$\begin{array}{l}\mathrm{NH}_{3}^{1} \\
(\mathrm{mg} / \mathrm{dl})\end{array}$} & \multicolumn{5}{|c|}{ VFA production ${ }^{2}$} \\
\hline & & & & & \multirow{2}{*}{$\begin{array}{l}\text { TVFA } \\
\text { (mmol/L) }\end{array}$} & $\mathrm{C}_{2}$ & $\mathrm{C}_{3}$ & $\mathrm{C}_{4}$ & \multirow[t]{2}{*}{$\mathrm{C}_{2}: \mathrm{C}_{3}$} \\
\hline & & & & & & \multicolumn{3}{|c|}{-_- $(\mathrm{mol} / 100 \mathrm{~mol})--$} & \\
\hline T1 & $60: 40$ & 0 & $\begin{array}{l}6.69 \\
\pm \\
0.02\end{array}$ & $\begin{array}{l}13.71 \\
\pm 1.01\end{array}$ & $\begin{array}{l}26.03 \pm \\
0.01\end{array}$ & $\begin{array}{l}65.91 \\
\pm 0.04\end{array}$ & $\begin{array}{l}21.72 \\
\pm 0.05\end{array}$ & $\begin{array}{l}12.51 \\
\pm 0.01\end{array}$ & $\begin{array}{l}3.31 \\
\pm \\
0.02\end{array}$ \\
\hline T2 & $60: 40$ & 2 & $\begin{array}{l}6.68 \\
\pm \\
0.01\end{array}$ & $\begin{array}{l}12.92 \\
\pm 0.09\end{array}$ & $\begin{array}{l}26.22 \pm \\
0.01\end{array}$ & $\begin{array}{l}65.02 \\
\pm 0.02\end{array}$ & $\begin{array}{l}22.71 \\
\pm 0.06\end{array}$ & $\begin{array}{l}12.42 \\
\pm 0.02\end{array}$ & $\begin{array}{l}2.92 \\
\pm \\
0.01\end{array}$ \\
\hline T3 & $60: 40$ & 4 & $\begin{array}{l}6.69 \\
\pm \\
0.01\end{array}$ & $\begin{array}{l}12.82 \\
\pm 0.09\end{array}$ & $\begin{array}{l}26.33 \pm \\
0.02\end{array}$ & $\begin{array}{l}64.61 \\
\pm 0.03\end{array}$ & $\begin{array}{l}23.24 \\
\pm 0.05\end{array}$ & $\begin{array}{l}12.22 \\
\pm 0.01\end{array}$ & $\begin{array}{l}3.01 \\
\pm \\
0.01\end{array}$ \\
\hline $\mathrm{T} 4$ & $60: 40$ & 6 & $\begin{array}{l}6.68 \\
\pm \\
0.01\end{array}$ & $\begin{array}{l}12.55 \\
\pm 1.01\end{array}$ & $\begin{array}{l}26.33 \pm \\
0.01\end{array}$ & $\begin{array}{l}63.91 \\
\pm 0.04\end{array}$ & $\begin{array}{l}23.91 \\
\pm 0.05\end{array}$ & $\begin{array}{l}12.23 \\
\pm 0.01\end{array}$ & $\begin{array}{l}2.82 \\
\pm \\
0.01\end{array}$ \\
\hline T5 & $60: 40$ & 8 & $\begin{array}{l}6.69 \\
\pm \\
0.01\end{array}$ & $\begin{array}{l}12.11 \\
\pm 1.01\end{array}$ & $\begin{array}{l}28.33 \pm \\
0.01\end{array}$ & $\begin{array}{l}64.12 \\
\pm 0.05\end{array}$ & $\begin{array}{l}24.43 \\
\pm 0.04\end{array}$ & $\begin{array}{l}11.41 \\
\pm 0.02\end{array}$ & $\begin{array}{l}2.72 \\
\pm \\
0.01\end{array}$ \\
\hline T6 & $60: 40$ & 10 & $\begin{array}{l}6.68 \\
\pm \\
0.01\end{array}$ & $\begin{array}{l}11.33 \\
\pm 0.08\end{array}$ & $\begin{array}{l}28.42 \pm \\
0.01\end{array}$ & $\begin{array}{l}63.11 \\
\pm 0.05\end{array}$ & $\begin{array}{l}25.72 \\
\pm 0.05\end{array}$ & $\begin{array}{l}11.23 \\
\pm 0.01\end{array}$ & $\begin{array}{l}2.71 \\
\pm \\
0.01\end{array}$ \\
\hline T7 & $60: 40$ & 12 & $\begin{array}{l}6.68 \\
\pm \\
0.01\end{array}$ & $\begin{array}{l}10.66 \\
\pm 0.04\end{array}$ & $\begin{array}{l}29.42 \pm \\
0.02\end{array}$ & $\begin{array}{l}63.53 \\
\pm 0.03\end{array}$ & $\begin{array}{l}26.51 \\
\pm 0.04\end{array}$ & $\begin{array}{l}10.02 \\
\pm 0.01\end{array}$ & $\begin{array}{l}2.72 \\
\pm \\
0.02\end{array}$ \\
\hline T8 & $40: 60$ & 0 & $\begin{array}{l}6.67 \\
\pm \\
0.01\end{array}$ & $\begin{array}{l}18.44 \\
\pm 0.07\end{array}$ & $\begin{array}{l}30.24 \pm \\
0.03\end{array}$ & $\begin{array}{l}65.91 \\
\pm 0.06\end{array}$ & $\begin{array}{l}22.62 \\
\pm 0.06\end{array}$ & $\begin{array}{l}11.51 \\
\pm 0.01\end{array}$ & $\begin{array}{l}3.01 \\
\pm \\
0.01\end{array}$ \\
\hline T9 & $40: 60$ & 2 & $\begin{array}{l}6.67 \\
\pm \\
0.01\end{array}$ & $\begin{array}{l}17.25 \\
\pm 0.05\end{array}$ & $\begin{array}{l}27.53 \pm \\
0.01\end{array}$ & $\begin{array}{l}62.62 \\
\pm 0.05\end{array}$ & $\begin{array}{l}26.13 \\
\pm 0.04\end{array}$ & $\begin{array}{l}11.31 \\
\pm 0.01\end{array}$ & $\begin{array}{l}2.71 \\
\pm \\
0.01\end{array}$ \\
\hline T10 & $40: 60$ & 4 & $\begin{array}{l}6.65 \\
\pm \\
0.01\end{array}$ & $\begin{array}{l}16.72 \\
\pm 0.06\end{array}$ & $\begin{array}{l}27.82 \pm \\
0.02\end{array}$ & $\begin{array}{l}61.32 \\
\pm 0.05\end{array}$ & $\begin{array}{l}27.92 \\
\pm 0.05\end{array}$ & $\begin{array}{l}10.82 \\
\pm 0.01\end{array}$ & $\begin{array}{l}2.52 \\
\pm \\
0.01\end{array}$ \\
\hline T11 & $40: 60$ & 6 & $\begin{array}{l}6.65 \\
\pm \\
0.01\end{array}$ & $\begin{array}{l}16.33 \\
\pm 0.04\end{array}$ & $\begin{array}{l}29.04 \pm \\
0.03\end{array}$ & $\begin{array}{l}61.41 \\
\pm 0.04\end{array}$ & $\begin{array}{l}28.02 \\
\pm 0.05\end{array}$ & $\begin{array}{l}10.61 \\
\pm 0.01\end{array}$ & $\begin{array}{l}2.51 \\
\pm \\
0.03\end{array}$ \\
\hline T12 & $40: 60$ & 8 & $\begin{array}{l}6.66 \\
\pm \\
0.01\end{array}$ & $\begin{array}{l}15.43 \\
\pm 0.05\end{array}$ & $\begin{array}{l}29.01 \pm \\
0.02\end{array}$ & $\begin{array}{l}61.11 \\
\pm 0.05\end{array}$ & $\begin{array}{l}28.51 \\
\pm 0.06\end{array}$ & $\begin{array}{l}10.42 \\
\pm 0.01\end{array}$ & $\begin{array}{l}2.32 \\
\pm \\
0.01\end{array}$ \\
\hline
\end{tabular}




\begin{tabular}{|c|c|c|c|c|c|c|c|c|c|}
\hline T13 & $40: 60$ & 10 & $\begin{array}{l}6.66 \\
\pm \\
0.01\end{array}$ & $\begin{array}{l}15.23 \\
\pm 0.04\end{array}$ & $\begin{array}{l}29.62 \pm \\
0.01\end{array}$ & $\begin{array}{l}60.53 \\
\pm 0.05\end{array}$ & $\begin{array}{l}29.22 \\
\pm 0.06\end{array}$ & $\begin{array}{l}10.31 \\
\pm 0.01\end{array}$ & $\begin{array}{l}2.23 \\
\pm \\
0.01\end{array}$ \\
\hline T14 & 40:60 & 12 & $\begin{array}{l}6.66 \\
\pm \\
0.01\end{array}$ & $\begin{array}{l}14.8 \pm \\
0.3\end{array}$ & $\begin{array}{l}30.11 \pm \\
0.22\end{array}$ & $\begin{array}{l}59.52 \\
\pm 0.03\end{array}$ & $\begin{array}{l}30.31 \\
\pm 0.04\end{array}$ & $\begin{array}{l}10.23 \\
\pm 0.01\end{array}$ & $\begin{array}{l}2.21 \\
\pm \\
0.02\end{array}$ \\
\hline \multicolumn{10}{|l|}{$P$-value } \\
\hline $\mathrm{R}: \mathrm{C}$ ratio & & & 0.001 & 0.01 & 0.001 & 0.001 & 0.001 & 0.01 & 0.001 \\
\hline RALP & & & 0.67 & 0.32 & 0.001 & 0.01 & 0.001 & 0.08 & 0.001 \\
\hline Interaction & & & 0.26 & 0.34 & 0.96 & 0.36 & 0.17 & 0.3 & 0.41 \\
\hline \multicolumn{10}{|c|}{${ }^{1} \mathrm{NH}_{3}-\mathrm{N}$ ammonia nitrogen } \\
\hline \multicolumn{10}{|c|}{$\begin{array}{l}2 \text { TVFA: total volatile fatty acid; VFA: volatile fatty acids; } \mathrm{C}_{2}: \text { acetic acid; } \mathrm{C}_{3}: \text { propionic acid; } \mathrm{C}_{4} \text { : butyric } \\
\text { acid; } \mathrm{C}_{2}: \mathrm{C}_{3}: \text { acetic acid to propionic acid ratio }\end{array}$} \\
\hline
\end{tabular}

Table 3, presents the findings of $\mathrm{pH}, \mathrm{NH}_{3}-\mathrm{N}$ and volatile fatty acid production. The rumen $\mathrm{pH}$ data remained similar, although slightly dropped when ratio of $\mathrm{R}: \mathrm{C}$ were lowered (6.69 to 6.56). Notable changes on rumen $\mathrm{NH}_{3}-\mathrm{N}$ when level of RALP increased for both $\mathrm{R}: \mathrm{C}$ at 60:40 and 40:60. Remarkable changes on rumen propionate production were obtained when level of RALP increased for both level of $\mathrm{R}: \mathrm{C}$, thus, lowering the $\mathrm{C}_{2}: \mathrm{C}_{3}$ ratio.

Table 4, shows rumen protozoal population and methane production. The rumen protozoal population ( $\times$ $10^{5} \mathrm{cell} / \mathrm{ml}$ ) and methane production $(\mathrm{mol} / \mathrm{L})$ were greatly influenced by the R:C and RALP supplementation and there were significant interactions for both factors. The methane $\left(\mathrm{CH}_{4}\right)$ production resulted in remarkable decline of $\mathrm{CH}_{4}$ for both $\mathrm{R}: \mathrm{C}$ and RALP supplementation but more dramatically for $R: C$ at 40:60, respectively. 
Table 4

Effect of red amaranth (Amaranth cruentus, L) leaf powder (RALP) supplementation on protozoal population and methane production

\begin{tabular}{|c|c|c|c|c|c|c|c|c|c|}
\hline \multirow[t]{2}{*}{ Treatments } & \multirow[t]{2}{*}{$\mathrm{R}: \mathrm{C}^{\mathrm{a}}$} & \multirow[t]{2}{*}{ RALP ${ }^{b}$} & \multicolumn{3}{|c|}{ Protozoa $\left(\times 10^{5} \mathrm{cell} / \mathrm{ml}\right)$} & \multicolumn{4}{|c|}{$\mathrm{CH}_{4}(\mathrm{~mol} / \mathrm{L})$} \\
\hline & & & $4 \mathrm{~h}$ & 8 & Mean & $4 \mathrm{~h}$ & 8 & 12 & Mean \\
\hline T1 & $60: 40$ & 0 & $\begin{array}{l}14.80 \\
\pm 0.35\end{array}$ & $\begin{array}{l}17.41 \\
\pm 0.40\end{array}$ & $\begin{array}{l}16.11 \\
\pm 0.12\end{array}$ & $\begin{array}{l}77.15 \\
\pm 1.02\end{array}$ & $\begin{array}{l}80.52 \\
\pm 1.11\end{array}$ & $\begin{array}{l}91.30 \\
\pm 0.97\end{array}$ & $\begin{array}{l}83.03 \\
\pm 0.12\end{array}$ \\
\hline T2 & $60: 40$ & 2 & $\begin{array}{l}13.51 \\
\pm 0.12\end{array}$ & $\begin{array}{l}16.14 \\
\pm 0.31\end{array}$ & $\begin{array}{l}14.80 \\
\pm 0.11\end{array}$ & $\begin{array}{l}75.42 \\
\pm 0.32\end{array}$ & $\begin{array}{l}79.01 \\
\pm 0.75\end{array}$ & $\begin{array}{l}90.43 \\
\pm 1.01\end{array}$ & $\begin{array}{l}81.64 \\
\pm 0.03\end{array}$ \\
\hline T3 & $60: 40$ & 4 & $\begin{array}{l}12.81 \\
\pm 0.42\end{array}$ & $\begin{array}{l}15.61 \\
\pm 0.11\end{array}$ & $\begin{array}{l}14.22 \\
\pm 0.24\end{array}$ & $\begin{array}{l}69.34 \\
\pm 0.32\end{array}$ & $\begin{array}{l}74.21 \\
\pm 0.45\end{array}$ & $\begin{array}{l}90.11 \\
\pm 0.92\end{array}$ & $\begin{array}{l}77.91 \\
\pm 0.04\end{array}$ \\
\hline $\mathrm{T} 4$ & $60: 40$ & 6 & $\begin{array}{l}11.82 \\
\pm 0.41\end{array}$ & $\begin{array}{l}14.20 \\
\pm 0.11\end{array}$ & $\begin{array}{l}13.01 \\
\pm 0.13\end{array}$ & $\begin{array}{l}68.04 \\
\pm 0.54\end{array}$ & $\begin{array}{l}67.82 \\
\pm 0.46\end{array}$ & $\begin{array}{l}87.63 \\
\pm 0.94\end{array}$ & $\begin{array}{l}74.52 \\
\pm 0.15\end{array}$ \\
\hline T5 & $60: 40$ & 8 & $\begin{array}{l}11.01 \\
\pm 0.71\end{array}$ & $\begin{array}{l}13.62 \\
\pm 0.71\end{array}$ & $\begin{array}{l}12.33 \\
\pm 0.03\end{array}$ & $\begin{array}{l}58.91 \\
\pm 0.43\end{array}$ & $\begin{array}{l}67.83 \\
\pm 0.35\end{array}$ & $\begin{array}{l}84.52 \\
\pm 0.34\end{array}$ & $\begin{array}{l}70.43 \\
\pm 0.12\end{array}$ \\
\hline T6 & $60: 40$ & 10 & $\begin{array}{l}10.50 \\
\pm 0.12\end{array}$ & $\begin{array}{l}12.91 \\
\pm 0.21\end{array}$ & $\begin{array}{l}11.71 \\
\pm 0.07\end{array}$ & $\begin{array}{l}56.62 \\
\pm 0.33\end{array}$ & $\begin{array}{l}63.51 \\
\pm 0.46\end{array}$ & $\begin{array}{l}83.60 \\
\pm 0.22\end{array}$ & $\begin{array}{l}67.92 \\
\pm 0.14\end{array}$ \\
\hline T7 & $60: 40$ & 12 & $\begin{array}{l}9.52 \pm \\
0.12\end{array}$ & $\begin{array}{l}11.90 \\
\pm 0.40\end{array}$ & $\begin{array}{l}10.72 \\
\pm 0.11\end{array}$ & $\begin{array}{l}54.30 \\
\pm 0.25\end{array}$ & $\begin{array}{l}61.02 \\
\pm 0.29\end{array}$ & $\begin{array}{l}83.32 \\
\pm 0.32\end{array}$ & $\begin{array}{l}66.24 \\
\pm 0.13\end{array}$ \\
\hline T8 & $40: 60$ & 0 & $\begin{array}{l}15.51 \\
\pm 0.13\end{array}$ & $\begin{array}{l}18.11 \\
\pm 0.41\end{array}$ & $\begin{array}{l}16.83 \\
\pm 0.12\end{array}$ & $\begin{array}{l}52.71 \\
\pm 0.54\end{array}$ & $\begin{array}{l}63.44 \\
\pm 0.43\end{array}$ & $\begin{array}{l}88.23 \\
\pm 0.33\end{array}$ & $\begin{array}{l}68.12 \\
\pm 0.13\end{array}$ \\
\hline T9 & $40: 60$ & 2 & $\begin{array}{l}15.03 \\
\pm 0.13\end{array}$ & $\begin{array}{l}17.62 \\
\pm 0.11\end{array}$ & $\begin{array}{l}16.34 \\
\pm 0.08\end{array}$ & $\begin{array}{l}50.75 \\
\pm 0.44\end{array}$ & $\begin{array}{l}59.22 \\
\pm 0.24\end{array}$ & $\begin{array}{l}87.24 \\
\pm 0.65\end{array}$ & $\begin{array}{l}65.72 \\
\pm 0.32\end{array}$ \\
\hline T10 & $40: 60$ & 4 & $\begin{array}{l}14.52 \\
\pm 0.11\end{array}$ & $\begin{array}{l}17.13 \\
\pm 0.11\end{array}$ & $\begin{array}{l}15.82 \\
\pm 0.12\end{array}$ & $\begin{array}{l}49.63 \\
\pm 0.23\end{array}$ & $\begin{array}{l}57.71 \\
\pm 0.46\end{array}$ & $\begin{array}{l}84.81 \\
\pm 0.35\end{array}$ & $\begin{array}{l}64.04 \\
\pm 0.30\end{array}$ \\
\hline T11 & $40: 60$ & 6 & $\begin{array}{l}13.81 \\
\pm 0.41\end{array}$ & $\begin{array}{l}16.43 \\
\pm 0.22\end{array}$ & $\begin{array}{l}15.11 \\
\pm 0.11\end{array}$ & $\begin{array}{l}42.55 \\
\pm 0.19\end{array}$ & $\begin{array}{l}56.63 \\
\pm 0.5\end{array}$ & $\begin{array}{l}77.31 \\
\pm 0.41\end{array}$ & $\begin{array}{l}58.83 \\
\pm 0.26\end{array}$ \\
\hline T12 & $40: 60$ & 8 & $\begin{array}{l}13.33 \\
\pm 0.40\end{array}$ & $\begin{array}{l}15.91 \\
\pm 0.41\end{array}$ & $\begin{array}{l}14.62 \\
\pm 0.14\end{array}$ & $\begin{array}{l}39.04 \\
\pm 0.46\end{array}$ & $\begin{array}{l}53.91 \\
\pm 0.52\end{array}$ & $\begin{array}{l}71.91 \\
\pm 0.44\end{array}$ & $\begin{array}{l}54.92 \\
\pm 0.42\end{array}$ \\
\hline T13 & $40: 60$ & 10 & $\begin{array}{l}12.32 \\
\pm 1.13\end{array}$ & $\begin{array}{l}14.91 \\
\pm 0.42\end{array}$ & $\begin{array}{l}13.61 \\
\pm 1.01\end{array}$ & $\begin{array}{l}35.72 \\
\pm 0.43\end{array}$ & $\begin{array}{l}52.21 \\
\pm 0.45\end{array}$ & $\begin{array}{l}68.10 \\
\pm 0.34\end{array}$ & $\begin{array}{l}52.04 \\
\pm 0.32\end{array}$ \\
\hline T14 & $40: 60$ & 12 & $\begin{array}{l}10.55 \\
\pm 0.15\end{array}$ & $\begin{array}{l}13.14 \\
\pm 0.21\end{array}$ & $\begin{array}{l}11.82 \\
\pm 0.98\end{array}$ & $\begin{array}{l}32.44 \\
\pm 0.25\end{array}$ & $\begin{array}{l}49.84 \\
\pm 0.33\end{array}$ & $\begin{array}{l}66.52 \\
\pm 0.21\end{array}$ & $\begin{array}{l}49.52 \\
\pm 0.31\end{array}$ \\
\hline \multicolumn{10}{|l|}{$P$-value } \\
\hline $\mathrm{R}: \mathrm{C}$ ratio & & & 0.07 & 0.07 & 0.01 & 0.05 & 0.001 & 0.001 & 0.001 \\
\hline RALP & & & 0.001 & 0.001 & 0.001 & 0.002 & 0.001 & 0.001 & 0.001 \\
\hline
\end{tabular}




\begin{tabular}{|c|c|c|c|c|c|c|c|c|c|}
\hline \multirow[t]{2}{*}{ Treatments } & \multirow[t]{2}{*}{$\mathrm{R}: \mathrm{C}^{\mathrm{a}}$} & \multirow[t]{2}{*}{ RALP } & \multicolumn{3}{|c|}{ Protozoa $\left(\times 10^{5} \mathrm{cell} / \mathrm{ml}\right)$} & \multicolumn{4}{|c|}{$\mathrm{CH}_{4}(\mathrm{~mol} / \mathrm{L})$} \\
\hline & & & $4 \mathrm{~h}$ & 8 & Mean & $4 \mathrm{~h}$ & 8 & 12 & Mean \\
\hline Interaction & & & 0.001 & 0.001 & 0.001 & 0.001 & 0.007 & 0.9 & 0.001 \\
\hline \multicolumn{10}{|c|}{$\begin{array}{l}\mathrm{CH}_{4} \text { : methane. } \\
\text { a Roughage : Concentrate ratio at } 60: 40 \text {, and } 40: 60 \% \text {. } \\
\text { b Levels of red amaranth leaf powder (RALP) supplementation at } 0,2,4,6,8,10 \text {, and } 12 \% \text { of total } \\
\text { substrate }\end{array}$} \\
\hline
\end{tabular}

\section{Discussion}

\section{Feed ingredients an feeding value}

Both roughage and concentrate sources can greatly impact to rumen characteristics when ingested into the rumen. Roughage and its fibrous characteristics will stimulate rumination and fermentation the activity of fibrolytic bacteria whilst carbohydrate sources will be additionally degraded by amylolytic bacteria. It has been reported that rumen $\mathrm{pH}$ should be buffered higher than 6.20 for efficient fiber degradation and to prevent incidence of subacute rumen acidosis (SARA) [32]. Ørskov [5] and Wanapat et al. [6] revealed the importance of roughage $(R)$ and concentrate $(C)$ ratio $(R: C)$ impacting on rumen $p H$, volatile fatty acid production and the variation of rumen microbiome especially fibrolytic bacteria namely $R$. albus, $R$. flavefaciens and Fibrobacter succinogenes, respectively $[6,33]$. Under this experiment, the two $R: C$ ratio used were 60:40 and 40:60 respectively, and the fermentation characteristics were influenced by the two ratio. Earlier work, Kang et al. [34] stated the impact of banana flower powder (BAFLOP) which contained phytonutrients and high concentration of minerals could lift up the rumen $\mathrm{pH}$ under high concentrate supplementation level both in the in vitro and in vivo feeding experiments. Under this trial, RALP which consisted of high crude protein $(15.80 \% \mathrm{CP}), 0.90 \%$ condensed tannins, $0.50 \%$ saponins, along with high concentrations of macro-minerals especially $\mathrm{Ca}, \mathrm{K}$, and $\mathrm{Mg}$. RALP could perform in the rumen similar to those of BAFLOP, as will be discussed in later section.

\section{In Vitro Gas Production And DM Degradability (\%)}

Under Table 2, the treatment combinations of two ratio of R:C, $60: 40$ and 40:60, along with level of RALP supplementation from $0,2,4,6,8,10$, and $12 \%$ total substrate and the fermentation gas kinetics and DM degradability (\%) are fully presented. The R:C ratio and RALP supplementation level did not significantly impact on $a$ and $b$, although there was an increasing trend for the $b$ values, when RALP supplementation level increased for both $R: C$ ratio. The $R: C$ ratio at 40:60 yielded higher $c$ constant than $R: C$ at $60: 40$. The $a+b$ data for both $\mathrm{R}: C$ ratio were increased $(P<0.01)$ by the RALP supplementation level and there were interactions $(P<0.01)$. The accumulated total production tended to be enhanced for both $\mathrm{R}: \mathrm{C}$ ratio but there were significant difference among treatments. 
In vitro DM degradability (\%) were measured both at 12 and $24 \mathrm{~h}$ of incubation. Higher RALP supplementation level remarkably increased the DM degradabilities for both $\mathrm{R}: \mathrm{C}$ more pronounced for $\mathrm{R}: \mathrm{C}$ at 40:60. The significant for both R:C and RALP interactions supplementation level were occurred $(P<$ 0.01 ). This occurrence could be due to more available carbohydrate for $R: C$ at $40: 60$ and from the incremental RALP supplementation with combined phytonutrients (condensed tannins and saponins) could enrich the fermentation process. Furthermore, higher concentration of minerals could help buffer $\mathrm{pH}$ especially for $\mathrm{R}: \mathrm{C}$ at 40:60. Aslam et al. [35] pointed out that the use of rumen buffer such as $\mathrm{NaHCO}_{3}$ could be beneficial when more concentration was fed. It was indicative that RALP supplementation could enhance the DM degradability, nevertheless, suitable level of supplementation be further investigated in in vivo trials.

\section{Rumen Fermentation Process}

Rumen fermentation end-products namely volatile fatty acids (VFAs) and ammonia-nitrogen $\left(\mathrm{NH}_{3}-\mathrm{N}\right)$ were synthesized by rumen microbiome. Russell abd Rychlik [36]; Wallace et al [37] and Huws et al. [38] have reiterated the close relationship of rumen microbiome and their fermentation efficiency. These fermentation end-products will serve as important substrates for the host-ruminants. Under this work, rumen $\mathrm{pH}$ not changed but maintained higher for $\mathrm{R}: \mathrm{C}$ at 60:40 as compared to 40:60 as the roughage could have attributed. It was notable that rumen $\mathrm{NH}_{3}-\mathrm{N}$ concentrations were higher for $\mathrm{R}: \mathrm{C}$ at $40: 60$ and were declined when RALP supplementation level were incremental for both R:C at 60:40 and 40:60, respectively and there were no significant interactions. Total VFAs $C_{2}, C_{3}, C_{4}$ and $C_{2}: C_{3}$ ratio were shown in Table 3. The $\mathrm{C}_{3}$ concentrations were elaborately shown to be enhanced by both R:C and RALP supplementation level, being more explicit for $\mathrm{R}: \mathrm{C}$ at 40:60 and with increasing supplementation level of RALP, whilst $\mathrm{C}_{2}: \mathrm{C}_{3}$ were greatly lowered $(P<0.01)$. Dietary source of RALP under this experiment has enormously supported the fermentation in which the beneficial outcomes were obtained as shown in Table 3. It was further speculated that level of RALP supplementation should be evaluated as they exhibited their supplementation effects.

Fermentation gas such as methane $\left(\mathrm{CH}_{4}\right)$ has been produced during anaerobic fermentation in the rumen. Johnson and Johnson [39] reported the loss of metabolizable energy in the form of $\mathrm{CH}_{4}$ up to $15 \%$ gross energy. Furthermore, $\mathrm{CH}_{4}$ is the one of the greenhouse gases and is 23 times more potent then $\mathrm{CO}_{2}$ equivalent. Hence, the mitigation of $\mathrm{CH}_{4}$ via rumen fermentation has been the major concern. Dietary manipulation in the rumen has been receiving more interests [40]. Under Table 4, the protozoal population enumerated at 4 and $8 \mathrm{~h}$ of incubation; were dramatically reduced for both R:C ratio and by higher RALP supplementation level and ther were greatly interative $(P<0.01)$. As shown by many researchers that the reduction of protozoal population in the rumen has a direct effect on methane production since some methanogens adhead on protozoa. In addition, the presence of phytonutrients contained in the feeds could also attribute to the mitigation of rumen methane. As presented under this work the rumen methane production were profoundly mitigated by increasing level of RALP supplementation for both R:C ratio at 
60:40 and 40:60, respectively. This result could very well support that RALP could be highly promising to be employed in feeding to ruminant to possible improve rumen fermentation efficiency and mitigate methane production.

\section{Conclusion}

Under this investigation, plants rich in phytonutrients and minerals such as red amaranth leaf powder (RALP) have a vital and promising rode to modulate rumen fermentation in maintaining $\mathrm{pH}$ enhancing propionate production mitigating methane production, as well as increasing DM degradability. RALP exhibits its potential role and deserves further in vivo trials investigation.

\section{Methods}

This study was approved by the Animal Care and Use Committee of Khon Kaen University.

\section{Experimental Design And Dietary Treatments}

The red amaranth seeds (Chia Tai seed company, Bangkok, Thailand) were bought from local market under the supervision and approval by corresponding author. The experiment was randomly assigned in factorial arrangement of two factors of roughage to concentrate and seven level of red amaranth leaf powder percentage of total substrate in a Completely randomized design (CRD). There were two factors, Factor A was two levels of roughage to concentrate ratio (R:C) at 60:40 and 40:60 of dietary substrate at $0.20 \mathrm{~g}$, while Factor B was level of red amaranth (Amaranthus cruentus, $L$ ) leaf powder (RALP) supplementation at $0,2,4,6,8,10$, and $12 \%$ of total dietary substrate.

Red amaranth leaf was collected from the plant after 25 days of growth. The red amaranth was planted on our experimental farm Khon Kaen University, Khon Kaen, Thailand. It was sun-dried, chopped and ground to achieve the $1 \mathrm{~mm}$ length. Standard chemical analyzed were employed to analyze for (DM), (OM), (CP) [18], neutral-detergent fiber (NDF), acid-detergent fiber (ADF) [19]. Additional chemical procedures on condensed tannins (CT) [20,21] as modified by Wanapat et al. [22] and saponins (SP) [23] were used. Macro minerals were determined using wet digestion (nitric-perchloric digestion), atomic absorption spectrophotometry (total $\mathrm{Ca}, \mathrm{K}, \mathrm{Mg}, \mathrm{Zn}, \mathrm{Fe}$ ) (model: analytic jena nova 350).

\section{Rumen And Substrate Inocula}

As a source of rumen inocula, two rumen-fistulated Holstein-Friesian dairy steer crossbreds ( $75 \%$ Holstein-Friesian and $25 \%$ Thai native breed, about $370 \mathrm{~kg}$ body weight) were used. Before the morning feeding, $1000 \mathrm{ml}$ of rumen fluid was collected from each animal and combined. The rumen fluid donors were fed with concentrate ( $14 \% \mathrm{DM}$ of $\mathrm{CP})$ at $0.5 \%$ of body weight $(\mathrm{BW})$ to maintain normal rumen 
ecology and rice straw was offered on ad libitum. The method used in this study was according to Menke et al. [24], as modified and described by Kang et al. [25].

\section{In Vitro Fermentation And Gas Production}

The in vitro fermentation kinetics and gas production of all treatment samples were run intervally starting from $1,2,4,6,8,12,24,48,72$, to $96 \mathrm{~h}$ post-incubation. Measurement of fermentation gas production was recorded at each time, $\mathrm{pH}$ was measured at 4,8 , and $12 \mathrm{~h}$ while the fluid was collected at 4 and $8 \mathrm{~h}$, and was divided into two parts. The first part of rumen fluid $(18 \mathrm{~mL})$ was collected and kept in a plastic bottle to which $2 \mathrm{~mL}$ of $1 \mathrm{M} \mathrm{H}_{2} \mathrm{SO}_{4}$ was added to discontinue fermentation process for later analyses of $\mathrm{NH}_{3}-\mathrm{N}$ by Kjeltec Auto 1030 Analyzer [18], volatile fatty acids $\left(\mathrm{C}_{2}, \mathrm{C}_{3}\right.$ and $\left.\mathrm{C}_{4}\right)$ using HPLC, Instruments by Water and Nova Pak model 600E; water mode 1484 UV detector; column nova Pak C18; column size $3.9 \mathrm{~mm} \times 300 \mathrm{~mm}$; mobile phase $10 \mathrm{mMH}_{2} \mathrm{PO}_{4}[\mathrm{pH} 2.5$ ] according to Samuel et al. [26] and the second portion of $1 \mathrm{ml}$ rumen fluid was collected and kept in a plastic bottle to which $9 \mathrm{ml}$ of $10 \%$ formalin solution for measuring of protozoal population using total direct count method by haemocytometer [27]. Methane production was determined from samples collected starting from 4, 8, to $12 \mathrm{~h}$ post-incubation internally using gas chromatography (Instruments by GC-17A System, Shimadzu; TCD detector; column shin carbon; column size $3 \mathrm{~m} \times 3 \mathrm{~mm}$, activated charcoal 60/80 mesh) [28]. The fermentation kinetic: $y=$

$a+b\left(1-e^{(-c t)}\right)$; where $a=$ gas production from immediately soluble fraction, $b=$ production of gas from the insoluble fraction, $c=$ constant gas production rate for the insoluble part $(b), t=$ time for incubation, $(a+$ $b)=$ the potential scope of gas production. $y=$ gas produced at the time " $t$." The in vitro dry matter degradability (\%) were calculated at both 12 and $24 \mathrm{~h}$ post-incubation were performed based on the Orskov and McDonal [29]. The in vitro dry matter degradability (\%) were calculated for both 12 and $24 \mathrm{~h}$ post-incubation.

\section{Statistical analysis}

All the obtained data were subjected to the General Linear Model (GLM) [30]. Differences among treatment means were compared by the Tukey's multiple comparison test [31]. Comparison between roughage to concentrate ratio, FMS supplementation and interactions were tested by orthogonal contrast.

\section{Abbreviations}

ADF: acid detergent fiber; BUN: blood urea nitrogen; BW: body weight; C: concentrate; CP: crude protein; $\mathrm{C}_{2}$ : acetate; $\mathrm{C}_{3}$ : propionate; $\mathrm{C}_{4}$ : butyrate; $\mathrm{CP}$ : condensed tannins; $\mathrm{DM}$ : dry matter; NDF: neutral detergent fiber; $\mathrm{NH}_{3}-\mathrm{N}$ : ammonia-nitrogen; $\mathrm{N}$ : nitrogen; OM: organic matter; RALP: red amaranth leaf powder; $\mathrm{R}$ : roughage; SP: saponins; TDN: total digestibility nutrient; VFA: volatile fatty acid

\section{Declarations}




\section{Ethics approval and consent to participate}

The experiment was officially agreed and approved by the Khon Kaen University Committee of Animal Care and Use for Research. The experimental cattle were provided by our research farm (TROFREC, KKU).

\section{Consent for publication}

Not applicable.

\section{Availability of data and materials}

All experimental data are responsible and available under the holding of the corresponding author.

\section{Competing interests}

The authors declare that they have no competing interests.

\section{Funding}

This research was supported by Tropical Feed Resources Research and Development Center (TROFREC), Department of Animal Science, Faculty of Agriculture, Khon Kaen University. Thailand Research Fund and International Research Network (TRF-IRN) TRF-IRN57W0002. KKU Scholarship for ASEAN and GMS Countries' Personnel. The Funding donor did not have role in the design of the study; collection, analysis, and interpretation of data; and in writing the manuscript.

\section{Authors' contributions}

MW designed the experiments; BV and TA. Conducted the animal experiments: BV and TA performed the analyses: MW, BV and TA. Wrote the manuscript. All authors reviewed and contributed to the manuscript. MW revised the final draft of manuscript. All authors read and approved the final manuscript.

\section{Acknowledgments}

The Tropical Feed Resources Research and Development Centre (TROFREC), Department of Animal Science, Faculty of Agriculture, Khon Kaen University, Thailand were gratefully acknowledged. Special gratitude are also extended to Thailand Research Fund under International Research Network (TRF-IRN), KKU Scholarship for ASEAN and GMS Countries' Personnel, TRF-IRN57W0002 and TRF-IRG598001, respectively. All graduate students especially Burarat Phesatcha, Maharach Matra, Pajaree Totakul are thankful for their assistances with samplings.

\section{Author information}

${ }^{1}$ Tropical Feed Resources Research and Development Center (TROFREC), Department of Animal Science, Faculty of Agriculture, Khon Kaen University, Khon Kaen 40002, Thailand 
${ }^{2}$ Department of Animal Science, Faculty of Agriculture and Technology Rajamangala University of Technology Isan Surin Campus, Surin 32000, Thailand

\section{References}

1. Hungate RE. The Rumen and its Microbes Academic Press NY. 1966.

2. Wolin MJ. A theoretical rumen fermentation balance. J Dairy Sci. 1960;43(10):1452-9.

3. Murphy MR, Baldwin RL, Koong LJ. Estimation of stoichiometric parameters for rumen fermentation of roughage and concentrate diets. J Anim Sci. 1982;55(2):411-21.

4. McAllister TA, Beauchemin KA, Alazzeh AY, Baah J, Teather RM, Stanford K. The use of direct fed microbials to mitigate pathogens and enhance production in cattle. Can J Anim Sci. 2011;91(2):193-211.

5. Ørskov ER, Fraser C, Gordon JG. Effect of processing of cereals on rumen fermentation, digestibility, rumination time, and firmness of subcutaneous fat in lambs. Br J Nutr. 1974;32(1):59-69.

6. Wanapat M, Gunun P, Anantasook N, Kang S. Changes of rumen $\mathrm{pH}$, fermentation and microbial population as influenced by different ratios of roughage (rice straw) to concentrate in dairy steers. $\mathrm{J}$ Agric Sci. 2014;152(4):675-85.

7. Herremans S, Vanwindekens F, Decruyenaere V, Beckers Y, Froidmont E. Effect of dietary tannins on milk yield and composition, nitrogen partitioning and nitrogen use efficiency of lactating dairy cows: A meta-analysis. J Anim Physiol Anim Nutr. 2020.

8. Castro-Montoya JM, Makkar HPS, Becker K. Chemical composition of rumen microbial fraction and fermentation parameters as affected by tannins and saponins using an in vitro rumen fermentation system. Can J Anim Sci. 2011;91(3):433-48.

9. Bodas R, Prieto N, García-González R, Andrés S, Giráldez FJ, López S. Manipulation of rumen fermentation and methane production with plant secondary metabolites. Anim Feed Sci Technol. 2012;176(1-4):78-93.

10. Kim JM, Park JA, Kim DH. Comparative proteomic analysis of chestnut blight fungus, Cryphonectria parasitica, under tannic-acid-inducing and hypovirus-regulating conditions. Canadian J Microbiol. 2012;58(7):863-71.

11. Singh IQBAL, Hundal JS, Wadhwa M, Lamba JS. Assessment of potential of some tannins and saponins containing herbs on digestibility of nutrients, fermentation kinetics and enteric methane production under different feeding systems: An in vitro study. Indian J Anim Sci. 2018;88:443-52.

12. Pulipati S, Babu PS, Naveena U, Parveen SR, Sumaya SK, Nausheen M. Determination of Total Phenolic, Tannin, Flavonoid Contents and Evaluation of Antioxidant Property of Amaranthus tricolor (L). Int J Pharm Phytoch Res. 2017;9(6):814-19.

13. Barba de la Rosa AP, Fomsgaard IS, Laursen B, Mortensen AG, Olvera-Martínez L, Silva-Sánchez C, Alberto Mendoza-Herrera J, González-Castañeda, De León-Rodríguez, A. Amaranth (Amaranthus 
hypochondriacus) as an alternative crop for sustainable food production: Phenolic acids and flavonoids with potential impact on its nutraceutical quality. J Cereal Sci. 2009;49(1):117-21.

14. Karamać M, Gai F, Longato E, Meineri G, Janiak MA, Amarowicz R, Peiretti PG. Antioxidant Activity and Phenolic Composition of Amaranth (Amaranthus caudatus) during Plant Growth. Antioxid. 2019;8(6):173.

15. Paśko P, Sajewicz M, Gorinstein S, Zachwieja Z. Analysis of selected phenolic acids and flavonoids in Amaranthus cruentus and Chenopodium quinoa seeds and sprouts by HPLC. Acta Chromatog. 2008;20(4):661-72.

16. Chairatanayuth P. Inclusion of amaranth crop residues in diet for cattle. Food Rev Int. 1992;8(1):15964.

17. Khandaker L, Akond AM, Ali MB, Oba S. Biomass yield and accumulations of bioactive compounds in red amaranth (Amaranthus tricolor $L$.) grown under different colored shade polyethylene in spring season. Sci Hortic. 2010;123(3):289-94.

18. AOAC (Association of Official Analytical Chemists). Official Methods of Analysis. 19th ed. Gaithersburg: Association of Official Analytical Chemists; 2012.

19. Van Soest PJ, Robertsonand JB, Lewis BA. Methods for dietary fiber neutral detergent fiber, and nonstarch polysaccharides in relation to animal nutrition. J Dairy Sci. 1991;74:3583-97.

20. Burns RE. Method for estimation of tannin in grain sorghum 1. Agron J. 1971;63(3):511-2.

21. Hagerman $A E$, Butler LG. Protein precipitation method for the quantitative determination of tannins. $J$ Agric Food Chem. 1978;26:809-12.

22. Wanapat $\mathrm{M}$, Poungchompu O. Method for estimation of tannin by vanillin- $\mathrm{HCl}$ method (A modified method of Burns, 1971). Department of Animal Science, Khon Kaen University, Khon Kaen,4002.2001.

23. Kwon JH, Belanger JM, Pare JJ, Yaylayan VA. Application of the microwave-assisted process $\left(\right.$ MAP $^{\mathrm{T}}$ ) to the fast extraction of ginseng saponins. Food Res Int. 2003;36(5):491-8.

24. Menke KH, Raab L, Salewski A, Steingass H, Fritz D, Schneider W. The estimation of the digestibility and metabolizable energy content of ruminant feedstuffs from the gas production when they are incubated with rumen liquor in vitro. J Agric Sci. 1979;92:217-22.

25. Kang S, Wanapat M, Viennasay B. Supplementation of banana flower powder pellet and plant oil sources on in vitro ruminal fermentation, digestibility, and methane production. Trop Anim Health Prod. 2016;48:673-1678.

26. Samuel M, Sagathevan S, Thomas J, Mathen G. An HPLC method for estimation of volatile fatty acids in ruminal fluid. Indian J Ani Sci. 1997;67:805-7.

27. Galyean ML. Laboratory procedure in animal nutrition research. Department of Animal and Life Science. USA: New Mexico State University; 1989. p. 188.

28. Sittijunda S, Reungsang A, O-thong S. Biohydrogen production from dual digestion pretreatment of poultry slaughterhouse sludge by anaerobic self-fermentation. Int J Hydro Energy. 2010;35:13427- 
34.

29. Ørskov ER, McDonal I. The estimation of protein degradability in the rumen from incubation measurements weighted according to rate of passage. J Agric Sci. 1979;92:499-503.

30. SAS (Statistical Analysis System). User's Guide: Statistic, Version 9.4th Edition. SAS Inst. Inc., Cary, NC. 2013.

31. Crichton N. Information point: Tukey Multiple Comparison test. Blackwell Science Ltd J Clin Nur. 1999;8:299-304.

32. Zebeli Q, Mansmann D, Steingass H, Ametaj BN. Balancing diets for physically effective fibre and ruminally degradable starch: A key to lower the risk of sub-acute rumen acidosis and improve productivity of dairy cattle. Livest Sci. 2010;127(1):1-10.

33. Koike S, Kobayashi Y. Development and use of competitive PCR assays for the rumen cellulolytic bacteria: Fibrobacter succinogenes, Ruminococcus albus and Ruminococcus flavefaciens. FEMS Microb Lett. 2001;204(2):361-6.

34. Kang $S$, Wanapat $M$, Cherdthorng A. Effect of banana flower powder supplementation as a rumen buffer on rumen fermentation efficiency and nutrient digestibility in dairy steers fed a highconcentrate diet. Anim Feed Sci Technol. 2014;196:32-41.

35. Aslam M, Tucker WB, Hogue JF, Vernon AK, Adams GD. Controlled Ruminal Infusion of Sodium Bicarbonate. 2. Effects of Dietary and Infused Buffer on Ruminal Milieu1. J Dairy Sci. 1991;74(10):3496-504.

36. Russell JB, Rychlik JL. Factors that alter rumen microbial ecology. Sci. 2001;292(5519):1119-22.

37. Wallace RJ, Rooke JA, McKain N, Duthie CA, Hyslop JJ, Ross DW, Watson M, Roehe R. The rumen microbial metagenome associated with high methane production in cattle. BMC Genom. 2015;16(1):839.

38. Huws SA, Creevey CJ, Oyama LB, Mizrahi I, Denman SE, Popova M, Muñoz-Tamayo R, Forano E, Waters SM, Hess M, Tapio I. Addressing global ruminant agricultural challenges through understanding the rumen microbiome: past, present, and future. Front Microb. 2018;9:2161.

39. Johnson KA, Johnson DE. Methane emissions from cattle. J Anim Sci. 1995;73(8):2483-92.

40. Hook SE, Steele MA, Northwood KS, Dijkstra J, France J, Wright ADG, McBride BW. Impact of subacute ruminal acidosis (SARA) adaptation and recovery on the density and diversity of bacteria in the rumen of dairy cows. FEMS Microbiol Ecol. 2011;78(2):275:284. 